\title{
Slow wind and magnetic topology in the solar minimum corona in 1996-1997
}

\author{
E. Antonucci ${ }^{1}$, L. Abbo ${ }^{1}$, and M. A. Dodero ${ }^{2}$ \\ 1 Istituto Nazionale di Astrofisica (INAF), Osservatorio Astronomico di Torino, Strada Osservatorio 20, \\ 10025 Pino Torinese, Italy \\ e-mail: [antonucci;abbo] ato.astro.it \\ 2 University of Torino, via P. Giuria 1, 10125 Torino, Italy
}

Received 23 January 2004 / Accepted 23 December 2004

\begin{abstract}
This study examines the physical conditions of the outer solar corona in order to identify the regions where the slow solar wind is accelerated and to investigate the latitudinal transition from slow to fast wind during the minimum of the solar cycle. The analysis is based on observations of six streamers obtained during the years of solar minimum, 1996 and 1997 , with the Ultraviolet Coronagraph Spectrometer (UVCS) onboard the Solar and Heliospheric Observatory (SOHO). The outflow velocity of the oxygen ions and the electron density of the coronal plasma are determined in altitude ranging from 1.5 to 3.5 solar radii $\left(R_{\odot}\right)$. The adopted diagnostic method, based on spectroscopic analysis of the O VI 1032 and $1038 \AA$ lines, fully accounts for the large expansion factor of the magnetic field lines expected in the regions surrounding the streamers. The analysis leads to the conclusion that the slow coronal wind is observed (i) in the region external to and running along the streamer boundary; and (ii) in the region above the streamer core beyond $2.7 R_{\odot}$, where the transition between closed and open magnetic field lines takes place and the heliospheric current sheet forms. Regions in the immediate vicinity of the streamer boundary can be identified with the edges of the large polar coronal holes that characterize solar minimum. Results point to gradual variations of the properties of a coronal hole from the streamer boundary to its polar core, most likely related to the topology of the coronal magnetic field.
\end{abstract}

Key words. Sun: corona - Sun: UV radiation - solar wind

\section{Introduction}

One of the primary aims of the Ultraviolet Coronagraph Spectrometer (Kohl et al. 1995) operating onboard the Solar and Heliospheric Observatory (SOHO), a joint ESA-NASA mission launched in 1995, is to identify the coronal sources of fast and slow wind by means of spectroscopic analysis of the ultraviolet line emission from the extended corona. The most intense lines formed in this region are mainly due to resonant scattering of the ultraviolet photons emitted from the chromosphere and transition region. In the expanding corona, emission by resonant scattering undergoes Doppler dimming. This effect allows the detection of radial outflows in the plane of the sky. Appropriate diagnostic techniques developed to interpret Doppler dimming (Antonucci 1998; Dodero et al. 1998; Li et al. 1998; Zangrilli et al. 2002; Antonucci et al. 2004) have allowed identification of the region of acceleration and signature of the acceleration mechanisms of the fast wind in polar coronal holes (Kohl et al. 1997, 1998; Antonucci 1999; Cranmer et al. 1999; Antonucci et al. 2000a, 2004). As for the slow wind, UVCS observations of the streamer belt at solar minimum have revealed an abundance anomaly (e.g.
Noci et al. 1997; Raymond et al. 1997; Marocchi et al. 2001; Ko et al. 2002; Uzzo et al. 2003) that might be related to the origin of the low-speed streams. Quiescent streamers show a more marked oxygen depletion in the core than in the streamer's bright lateral branches (Noci et al. 1997; Raymond et al. 1997). Noci et al. propose relating this dimming to the existence of open magnetic field lines inside magnetically complex streamers, thus channelling slow speed plasma into the heliosphere. On the other hand, Raymond et al. (1997) propose gravitational settling to explain oxygen depletion in the streamer core. Marocchi et al. (2001) point out that both gravitational settling acting inside sub-streamers and outflows along open field lines between sub-streamers may coexist. Results by Frazin et al. (2003) would, however, exclude significant outflow velocities in the streamer core in contrast with the Noci et al. interpretation. Direct observations of slow wind in the proximity of streamers were recently carried out by Strachan et al. (2002) and Abbo \& Antonucci (2002a). Strachan et al. estimate the outflow velocity via classical Doppler dimming analysis based on the ratio of O VI line emissivities (Noci et al. 1987), provided the electron density is independently derived from the visible light emission observed with the 
Table 1. Coronal streamers observed with UVCS-SOHO.

\begin{tabular}{cccc}
\hline \hline Date & $\begin{array}{c}\text { Polar angle } \\
\Theta\left({ }^{\circ}\right)\end{array}$ & $\begin{array}{c}\text { Altitude range } \\
\left(R_{\odot}\right)\end{array}$ & $\begin{array}{c}\text { Time interval } \\
(\mathrm{UT})\end{array}$ \\
\hline 19 Aug. 1996 & 135 & $1.5-3.8$ & $17: 49: 12-04: 14: 36$ \\
22 Aug. 1996 & 45 & $1.5-3.8$ & $20: 55: 24-05: 55: 58$ \\
30 Aug. 1996 & 225 & $1.5-3.8$ & $16: 27: 47-02: 37: 36$ \\
01 Sep. 1996 & 285 & $1.5-3.8$ & $16: 50: 04-01: 50: 23$ \\
30 Apr. 1997 & 288 & $1.5-3.5$ & $20: 58: 33-06: 41: 28$ \\
05 May 1997 & 72 & $1.5-3.5$ & $18: 31: 37-01: 34: 08$ \\
\hline
\end{tabular}

C2 coronagraph of LASCO (SOHO). The analysis is thus restricted to the region above $2 R_{\odot}$, the lower limit of the LASCO C2 field of view. They find the insurgence of outflows between 3.6 and $4.1 R_{\odot}$ along the streamer axis. The existence of slow wind at $80-100 \mathrm{~km} \mathrm{~s}^{-1}$ outside the streamer is determined only at a fixed height, $2.3 R_{\odot}$. Abbo \& Antonucci (2002a) adopt an improved version of the Doppler dimming technique that derives both electron density and outflow velocity of the expanding corona from the O VI 1032, 1037 line intensities, given constraint on the geometry of the flow tube connecting corona and heliosphere (Antonucci et al. 2004). This technique extends study of the dynamics of the corona below $2 R_{\odot}$ in a region where space coronagraphs typically fail to measure the polarized visible light. Abbo and Antonucci observed coronal expansion all along the streamer boundary, above $1.7 R_{\odot}$, and above the streamer cusp, approximately at $2.3-2.7 R_{\odot}$. Slow velocities from 50 to $80 \mathrm{~km} \mathrm{~s}^{-1}$ were observed. Where the plasma is expanding, higher levels of kinetic energy of the O VI ions are found, indicating energy deposition occurring along the open field lines just outside streamers. In the present paper we investigate the slow wind properties by adopting, in the Doppler dimming analysis, large expansion factors of the flow tubes expected in the coronal regions running along the streamer boundary (Wang \& Sheeley 1990). The streamers selected for this study were observed during the last solar minimum (1996-1997), when the two regimes of fast and slow wind were clearly detected in the heliosphere with Ulysses and the coronal configuration was close to a dipole (e.g. Phillips et al. 1995; McComas et al. 1998). The present study also investigates the transition between the two wind regimes in corona to verify if it is as abrupt as observed in the heliosphere, where these regimes are characterized by quite different conditions of flow velocity, composition, and chargestate (e.g. von Steiger 2000). Preliminary results of the present study are reported by Abbo et al. (2003) and Abbo (2002).

\section{Streamer observations in the years of minimum activity}

In order to investigate the source and acceleration regions of the slow solar wind, we select six streamers observed at high spectral resolution with the Ultraviolet Coronagraph Spectrometer (UVCS) during the recent solar minimum, more precisely: in 1996 on August 19, 22, 30, and on September 1, and in 1997 on April 30 and on May 5 (Table 1). The UVCS field of view (FOV) is superposed on the images of selected streamers. The composite images were obtained with the $\mathrm{C} 1$ and $\mathrm{C} 2$ visible light LASCO coronagraphs (Fig. 1), while the disk images were obtained with the EIT/SOHO in the Fe XII $195 \AA$ line. UVCS scans covered a broad region of the corona above $1.5 R_{\odot}$ and crossed at least one of the streamer boundaries, in order to cover both the streamer, or part of it, and part of the adjacent coronal hole, thereby encompassing the transition region between the closed and open field lines. Analysis is based on the O VI doublet at 1032 and $1038 \AA$ and the HI Lyman $\alpha$ line at $1216 \AA$. The slit of the UVCS is centered on the radial direction at a given position angle $\Theta$ (in degrees, counterclockwise from the North pole) and defines the instantaneous field of view (IFOV) of the observation. Streamers are observed by positioning the slit at increasing heights from $1.5 R_{\odot}$ to $3.5-3.8 R_{\odot}$. The position angle, altitude range of the UVCS, total field of view, and both initial and final times of the observations are reported in Table 1. On August 19, 22, 30 and September 1, 1996, the corona was observed from $1.5 R_{\odot}$ to $3.8 R_{\odot}$, with the slit successively positioned at the following heights: $1.5,1.6,1.7,1.8,1.9,2.0$, $2.1,2.3,2.5,2.7,2.9,3.3$ and $3.8 R_{\odot}$. The full scan of the streamer lasted approximately $10 \mathrm{~h}$. The corona was scanned from $1.5 R_{\odot}$ to $3.5 R_{\odot}$ on the days April 30 and May 5, 1997 for approximately $8 \mathrm{~h}$, and the observed heights were: $1.5,1.7,1.9,2.2,2.5,3.0$ and $3.5 R_{\odot}$. The instantaneous field of view in most of the observations was chosen to be (37 $\operatorname{arcmin} \times 14 \operatorname{arcsec})$ slit length and width, respectively, for the observations on August 19, 22, 30 and September 1, 1996 and (38 $\operatorname{arcmin} \times 14 \operatorname{arcsec})$ on April 30 and May 5, 1997. Elemental areas $(56 \operatorname{arcsec} \times 14$ arcsec) wide were observed along the IFOV on August 19, 22, 30 and September 1, 1996, and (21 $\operatorname{arcsec} \times 14 \operatorname{arcsec})$ wide on April 30 and May 5, 1997. The spectral resolution is $0.18 \AA$, as determined by a slit width of 14 arcsec. Observations beyond $2.9 R_{\odot}$, performed on August 22, 30 and September 1, 1996 are discarded since the spectrometer slit was opened to 84 arcsec, thus degrading the spectral resolution to $1.08 \AA$. Exposure time was chosen to increase with height to compensate for the concurrent decrease in emission. In order to enhance the statistics further, nearby exposures are summed resulting in the following average heliodistances: $1.6,1.85,2.15,2.6$, and $3.5 R_{\odot}$ for the streamers of August 19, 22, 30 and September 1, 1996; 1.8, 2.3, and $3.3 R_{\odot}$ and $1.7,2.3$, and $3.3 R_{\odot}$ for the observations performed on April 30, 1997 and on May 5, 1997, respectively (Fig. 1). 

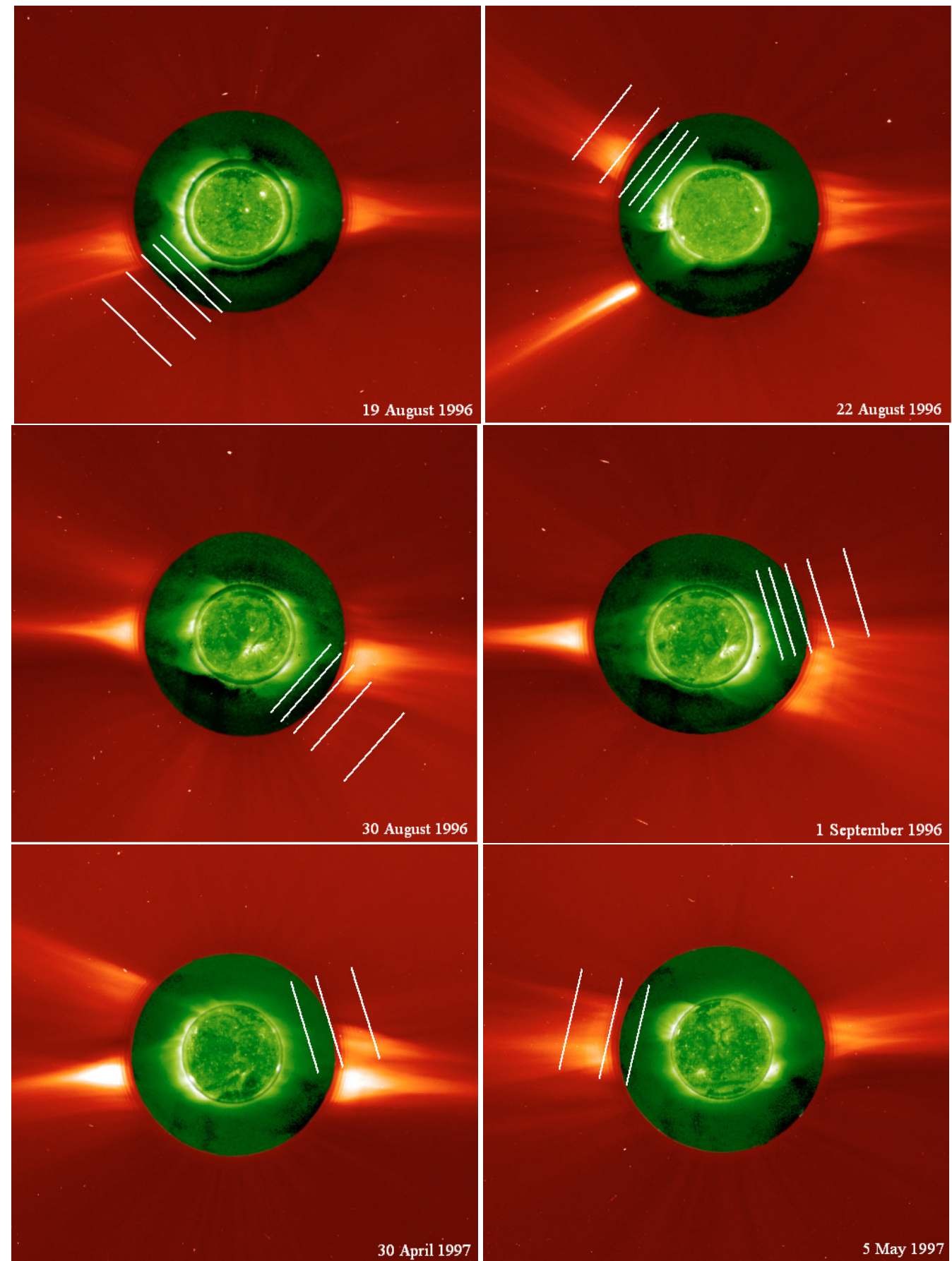

Fig. 1. Composite images of the Sun. The disk is imaged with EIT/SOHO in the Fe XII $195 \AA$ line. The limb images are obtained with the LASCO/SOHO C1/C2 coronagraphs. The slit (IFOV) of UVCS is superposed to the coronal images.

\section{Data analysis method}

Solar minimum streamers were first modelled by Pneuman \& Kopp (1971) by considering the steady expansion of a corona overlying a spherical surface characterized by a dipolar magnetic field. The minimum solar atmosphere indeed appears to be very similar to a dipole stretched by the coronal expansion and thus to be formed by an equatorial annular structure where the plasma is confined by the closed field that is surrounded by the open field lines of the large polar coronal holes. The first step in the analysis consists then in identifying the streamer boundary on the basis of the ultraviolet emission detected with the UVCS. When correctly defined, the boundary coincides with the streamer-coronal hole interface. The second step consists in determining physical and dynamical characteristics of the coronal plasma in the two regions separated by the boundary, namely, the bright streamer and the elongated dim region externally adjacent to the streamer boundary. Due to the relative position of the scan and streamer axis, just one boundary can be identified in most observations. In few cases, further out, the slit covers only the region outside the boundary. 


\subsection{Streamer boundary definition}

We define first the streamer boundary on the basis of latitudinal variation in the emission, and then we derive the plasma physical parameters from the core of the streamer outwards. From the point of view of the magnetic configuration, the streamercoronal hole interface corresponds to the transition between closed field lines, that confine the coronal plasma and the open magnetic fields. In the absence of direct measurement of the coronal magnetic field, the streamer boundary can be defined according to several other physical parameters (Dobryzcka et al. 1999; Zurbuchen et al. 2001; Abbo \& Antonucci 2002b). In coronal images the streamer/coronal hole interface is observed as a dimming of visible light and ultraviolet emission (e.g. Dobryzcka et al. 1999). The UVCS data show that at the streamer boundary, decrease in the ultraviolet intensity of lines such as O VI 1032 and 1038 is correlated with a significant increase in the width of the oxygen ion velocity distribution along the line-of-sight observed as OVI line broadening (Antonucci et al. 1997a,b). The O VI line width becomes extremely large as it approaches the polar corona (e.g. Antonucci et al. 1997b; Kohl et al. 1997). This latitudinal dependence of the O VI line width was confirmed by Dobryzcka et al. (1999) and Zangrilli et al. (1999), who analyzed the corona below $2.25 R_{\odot}$. In the proximity of the interface between closed and open field lines we also expect insurgence of coronal outflows, as shown by the 0.5 contour level of the ratio of the O VI 1038 and 1032 lines, which corresponds to $100 \mathrm{~km} \mathrm{~s}^{-1}$ in speed and roughly outlines coronal streamers (Antonucci et al. 1997b; Giordano et al. 1997; Habbal et al. 1997). The streamer boundary can thus be defined based on at least three parameters: (i) the intensity of the coronal emission; (ii) the kinetic temperature of the oxygen ions; and (iii) the expansion velocity of the coronal plasma. In order to quantify these parameters, we consider the contour levels corresponding to: (i) the $1 / e$ decrease in the O VI 1032 and 1038 emission (in our case relative to the maximum value observed along the UVCS instantaneous field of view); (ii) the maximum gradient of the kinetic temperature of oxygen ions as a function of position angle $\Delta T / \Delta \Theta$ derived from line broadening; (iii) the O VI 1038, 1032 line intensity ratio $\rho=0.5$, corresponding approximately to an outflow velocity $w$ of about $100 \mathrm{~km} \mathrm{~s}^{-1}$. It turns out that the last two contours $(\Delta T / \Delta \Theta)_{\max }$ and $w \sim 100 \mathrm{~km} \mathrm{~s}^{-1}$ are both running externally to the $1 / e$ emission boundary by approximately $\leq 10$ degrees and $\leq 20$ degrees, respectively (Abbo \& Antonucci 2002b). In this analysis the streamer border is, therefore, chosen to correspond to the inner contour level as defined in terms of the $1 / e$ value of the O VI 1032 maximum emission along the slit. Figure 2 gives an example of streamer boundary determination, while Table 2 reports the spatial parameters of the streamers and their adjacent dim regions. From $1.5 R_{\odot}$ to $2.7 R_{\odot}$, the width of the dim regions outside the streamer boundary turns out to be on the average about 15 degrees. It is worthwhile to note that, when defined in terms of the $1 / e$ value of HI Lyman $\alpha$ maximum emission along the slit, the streamer boundary remains roughly the same. Counts of the O VI 1032 and 1038 lines are separately integrated over the regions divided by the boundary/ies, namely, streamer and adjacent regions. Counts are then corrected for stray light and transformed to intensity $I(\lambda)$ by applying the standard radiometric calibration (Gardner et al. 1996). The spectral line profiles $I(\lambda)$ are fitted with a Gaussian function, that represents the solar line profile, convolved with a Voigt curve and an appropriate function that account for the instrumental broadening and the width of the spectrometer slit, respectively. The function resulting from the convolution is added to a background linearly dependent on wavelength. The best fit is obtained by applying the least square method using the following as adjustable parameters: peak intensity $I_{0}$, standard deviation $\sigma_{\lambda}$, and mean wavelength $\lambda_{0}$ of the solar profile, and slope and intercept of the background. The parameters defining the instrumental Voigt function and the corrections for stray light are those derived according to the method in Giordano (1999). The line intensity is obtained by integrating over the solar line profile. Standard deviation $\sigma_{\lambda}$ can be expressed in terms of the kinetic temperature of coronal ions $T_{\mathrm{k}}$ along the line of sight: $\sigma_{\lambda}=\frac{\lambda_{0}}{c} \sqrt{\frac{k_{\mathrm{B}} T_{\mathrm{k}}}{m_{\mathrm{p}} A}}$, where $c$ is the speed of light, $k_{\mathrm{B}}$ the Boltzmann constant, $m_{\mathrm{p}}$ the proton mass, and $A$ the ion mass number. The kinetic temperature then measures the width of the velocity distribution of the oxygen ions along the line of sight.

\subsection{Spectroscopic diagnostics}

Both electron density and outflow velocity of the coronal plasma inside streamers and in the regions running along the streamer boundaries are derived by analyzing the ultraviolet lines emitted at $1031.91 \AA$ and $1037.61 \AA$ by the O VI ions. The two lines are bright in streamers and sufficiently intense even in the dim regions of the extended corona surrounding streamers. The diagnostic method we use fully accounts for the Doppler dimming effect in those regions where the solar wind is accelerated and includes the simpler case of a static corona (Antonucci et al. 2004). This method can be applied throughout the extended corona, even below $2 R_{\odot}$ where visible light observations are lacking, thereby allowing us to obtain unique information on the dynamics of the corona.

Doppler dimming derives from the fact that, in the frame of reference of the expanding corona, the exciting spectrum originating in the transition region is red-shifted relative to the coronal absorbing profile. The dimming factor $\Phi(\delta \lambda)$ is defined as the integral of the product of the exciting intensity $I_{\mathrm{ex}}(\lambda)$ and the normalized coronal absorption profile $\Psi(\lambda)$ along the direction of the incident radiation, expressed as a function of wavelength $\lambda$

$\Phi(\delta \lambda)=\int_{0}^{+\infty} I_{\mathrm{ex}}(\lambda-\delta \lambda, \boldsymbol{n}) \Psi\left(\lambda-\lambda_{0}\right) \mathrm{d} \lambda$,

where $\lambda_{0}$ is the reference wavelength, $\delta \lambda$ the shift of the disk spectrum introduced by the radial outflow velocity $w$ of the coronal absorbing ions along direction $\boldsymbol{n}$ :

$\delta \lambda=\frac{\lambda_{0}}{c} \boldsymbol{w} \cdot \boldsymbol{n}$.

When the red-shift $\delta \lambda$ of the exciting spectrum is sufficiently large, pumping of the O VI 1037.61 $\AA$ line by the two nearby 

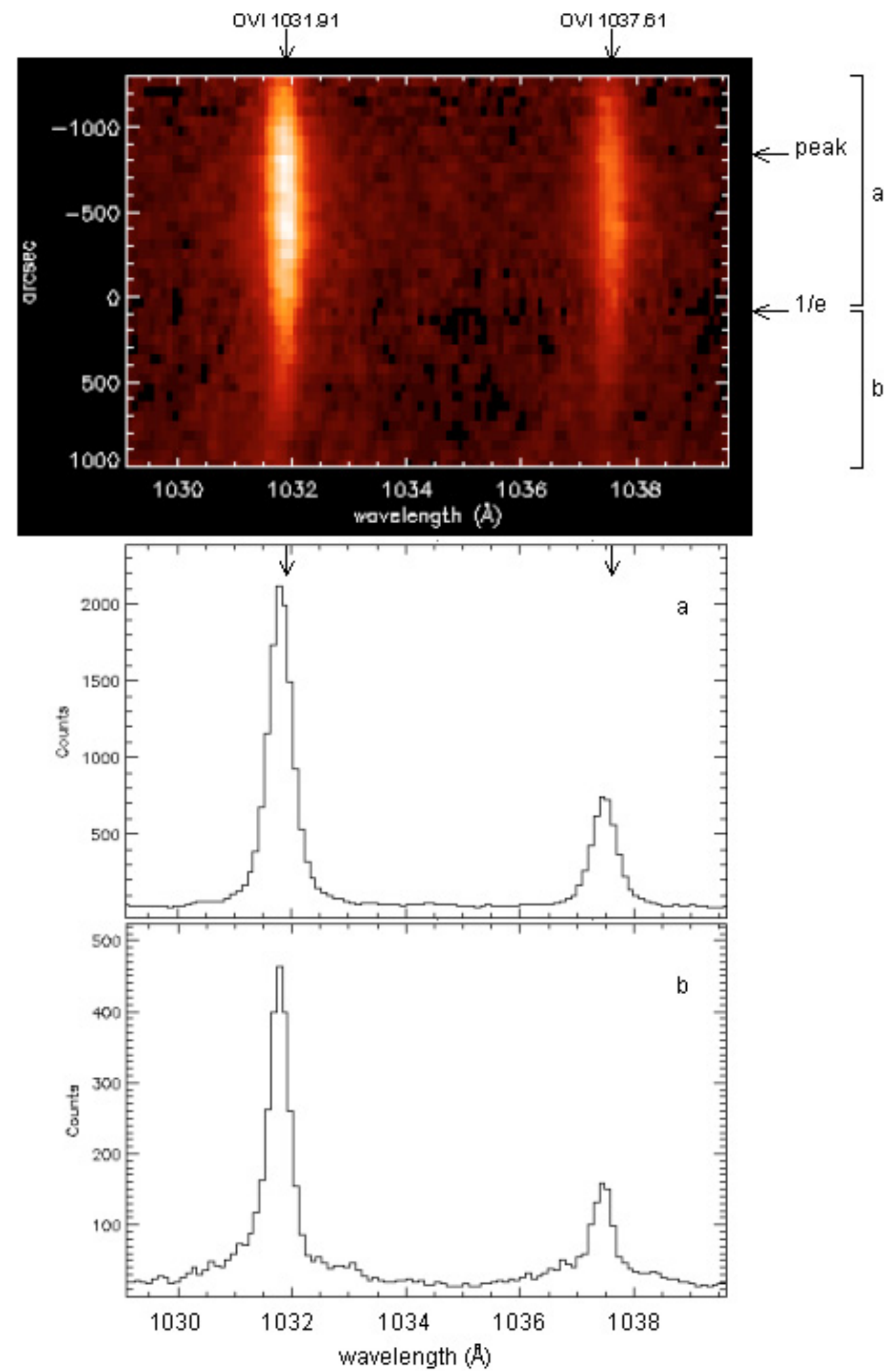

Fig. 2. Top panel: intensity of the O VI 1031.91, $1037.61 \AA$ lines along the slit of the UVCS, i.e., the instantaneous field of view (vertical axis) across the radial direction at $1.85 R_{\odot}$ on August 30, 1996. The horizontal axis is along the spectral dispersion; the wavelength range, from $1029.0 \AA$ to $1039.5 \AA$, includes the O VI doublet. Vertical arrows mark the laboratory wavelengths of the O VI lines. The labels $a$ and $b$ indicate the regions corresponding to the bright streamer and the dimmer region external to the streamer boundary, respectively. The horizontal arrows correspond to the intensity peak of the O VI 1032 line and the streamer boundary, defined as the 1/e 1032 O VI peak intensity level. Bottom panels: profiles of the O VI lines averaged over the regions $a$ and $b$, as indicated by the corresponding labels in the top panel. 
Table 2. Position angles (in degrees) of the boundary $\Theta_{\mathrm{b}}$ within each IFOV, as a function of height (in solar radii, $R_{\odot}$ ). $\Theta_{\text {int }}$ and $\Delta \Theta_{\text {int }}$ define the central position and half width of the streamer extension defined by either the two boundaries or one boundary and one edge of the slit. $\Theta_{\text {ext }}$ and $\Delta \Theta_{\text {ext }}$ define the central position and half width of the external region, delimited by the boundary and the other edge of the slit.

\begin{tabular}{|c|c|c|c|c|c|c|c|}
\hline \multicolumn{8}{|c|}{19 August 1996} \\
\hline Height & $\Theta_{\mathrm{b}}$ & Height & $\Theta_{\text {int }}$ & $\Delta \Theta_{\text {int }}$ & Height & $\Theta_{\text {ext }}$ & $\Delta \Theta_{\mathrm{ext}}$ \\
\hline 1.52 & 139 & 1.61 & 119 & 20 & 1.74 & 161 & 22 \\
\hline 1.82 & 133 & 1.91 & 118 & 15 & 1.98 & 155 & 22 \\
\hline 2.16 & 129 & 2.24 & 119 & 10 & 2.27 & 150 & 21 \\
\hline \multirow[t]{2}{*}{2.74} & 122 & 2.80 & 118 & 4 & 2.75 & 143 & 21 \\
\hline & & & & & 3.50 & 136 & 35 \\
\hline \multicolumn{8}{|c|}{22 August 1996} \\
\hline Height & $\Theta_{\mathrm{b}}$ & Height & $\Theta_{\text {int }}$ & $\Delta \Theta_{\text {int }}$ & Height & $\Theta_{\mathrm{ext}}$ & $\Delta \Theta_{\mathrm{ext}}$ \\
\hline 1.55 & 34 & 1.61 & 59 & 25 & 1.67 & 21 & 13 \\
\hline 1.83 & 38 & 1.91 & 59 & 21 & 1.93 & 26 & 12 \\
\hline 2.15 & 40 & 2.27 & 61 & 21 & 2.24 & 29 & 11 \\
\hline 2.67 & 49 & 2.78 & 60 & 11 & 2.69 & 39 & 10 \\
\hline 3.48 & 50 & 3.59 & 59 & 9 & 3.48 & 42 & 8 \\
\hline \multicolumn{8}{|c|}{30 August 1996} \\
\hline Height & $\Theta_{b}$ & Height & $\Theta_{\text {int }}$ & $\Delta \Theta_{\text {int }}$ & Height & $\Theta_{\mathrm{ext}}$ & $\Delta \Theta_{\text {ext }}$ \\
\hline 1.52 & 219 & 1.58 & 246 & 27 & 1.64 & 204 & 15 \\
\hline 1.82 & 221 & 1.92 & 244 & 23 & 1.91 & 208 & 13 \\
\hline 2.18 & 228 & 2.38 & 245 & 17 & 2.21 & 213 & 15 \\
\hline 2.80 & 243 & 2.91 & 248 & 5 & 2.70 & 228 & 15 \\
\hline \multicolumn{8}{|c|}{1 September 1996} \\
\hline Height & $\Theta_{\mathrm{b}}$ & Height & $\Theta_{\text {int }}$ & $\Delta \Theta_{\text {int }}$ & Height & $\Theta_{\mathrm{ext}}$ & $\Delta \Theta_{\text {ext }}$ \\
\hline 1.59 & 302 & 1.60 & 275 & 27 & 1.81 & 316 & 14 \\
\hline 1.83 & 292 & 1.89 & 273 & 19 & 2.02 & 309 & 17 \\
\hline 2.14 & 285 & 2.22 & 271 & 14 & 2.29 & 303 & 18 \\
\hline 2.68 & 280 & 2.75 & 272 & 8 & 2.77 & 297 & 17 \\
\hline \multicolumn{8}{|c|}{30 April 1997} \\
\hline Height & $\Theta_{\mathrm{b}}$ & Height & $\Theta_{\mathrm{int}}$ & $\Delta \Theta_{\text {int }}$ & Height & $\Theta_{\mathrm{ext}}$ & $\Delta \Theta_{\mathrm{ext}}$ \\
\hline 1.76 & 302 & 1.72 & 287 & 15 & 1.95 & 316 & 14 \\
\hline 2.36 & 284 & 2.43 & 274 & 10 & 2.47 & 302 & 18 \\
\hline 3.30 & 278 & 3.35 & 275 & 3 & 3.33 & 295 & 17 \\
\hline \multicolumn{8}{|c|}{5 May 1997} \\
\hline Height & $\Theta_{\mathrm{b}}$ & Height & $\Theta_{\mathrm{int}}$ & $\Delta \Theta_{\text {int }}$ & Height & $\Theta_{\mathrm{ext}}$ & $\Delta \Theta_{\text {ext }}$ \\
\hline 1.84 & 61 & 1.87 & 81 & 20 & 1.93 & 52 & 9 \\
\hline 2.36 & 70 & 2.45 & 85 & 15 & 2.43 & 59 & 11 \\
\hline 3.27 & 77 & 3.38 & 86 & 9 & 3.29 & 66 & 11 \\
\hline
\end{tabular}

chromospheric C II lines at $1037.02 \AA$ and $1036.34 \AA$ is induced (Noci et al. 1987; Dodero et al. 1998 and Li et al. 1998). However partial pumping can take place even in the static case, $w=0$, when the absorbing profiles are affected by large broadenings, as occurs in the open magnetic field line regions of the polar solar minimum corona. The exciting spectrum along the direction of the incident radiation is given by the disk intensities of the O VI doublet lines derived by Raymond et al. (1997) and of the C II 1037 and 1036 lines measured by Wilhelm et al. (1998). The disk intensities are corrected to account for limb brightening of the transition region O VI lines by considering a non-uniform distribution over the disk that approximates the 
one observed with the SUMER spectrometer (A. H. Gabriel \& J.-C. Vial, private communication). This aspect of the analysis, although more important in the inner corona, cannot be neglected in the extended corona.

The density of the coronal plasma averaged along the lineof-sight is proportional to the ratio of the collisional $I_{\mathrm{c}}$ and radiative $I_{\mathrm{r}}$ components of a line (e.g. the O VI 1032 line) according to the equation

$\left\langle n_{\mathrm{e}}\right\rangle \sim \frac{I_{\mathrm{c}}}{I_{\mathrm{r}}} \frac{h v_{0} B_{12}}{\left\langle q_{\mathrm{coll}}\right\rangle}\left\langle\int_{\Omega} F(w) p(\varphi) \mathrm{d} \omega\right\rangle$,

where $F(w)=\frac{\lambda^{2}}{c} \Phi(\delta \lambda)$ and, therefore, depends on the mean velocity $w$ of the coronal ions. Function $p(\varphi)$ takes into account the geometry of the scattering process, where $\varphi$ is the angle between $\boldsymbol{n}$ and the l.o.s., d $\omega$ the infinitesimal solid angle around $n$, and $\Omega$ the solid angle subtended by the solar disk at the point of scattering. $B_{12}$ is the Einstein coefficient for absorption. Integration of the emission due to photons approaching the point of scattering along different directions within the solid angle $\Omega$ is essential in order to fully account for the fact that the Doppler shift and the absorbing profiles (for bi-maxwellian distributions of the absorbing coronal ions) depend on the direction of the incident radiation. Departures from ionization equilibrium do not affect the density results, since both the collisional and radiative component are proportional to the ion column density and, therefore, to the oxygen ionization fraction. The radiative and collisional components of each line can be separated by taking into account: (i) the observed line intensities of the two OVI lines (indicated as $a$ and $b$ ); (ii) sum of the two components; and (iii) the ratios $R_{1}$ and $R_{2}$ of the radiative and collisional components of the two lines, respectively. $R_{2}$ depends only on the atomic parameters of the lines and the electron temperature. Ratio $R_{1}$ is a function of the ratio of the Doppler dimming factors of the lines (e.g. Antonucci et al. 2004):

$\frac{I_{\mathrm{r}, a}}{I_{\mathrm{r}, b}}=R_{1} \sim \frac{b_{a}}{b_{\mathrm{b}}} \frac{B_{12, a}}{B_{12, b}} \frac{\lambda_{0, a}}{\lambda_{0, b}} \frac{\left\langle\int_{\Omega} \Phi_{a}(\delta \lambda) p(\varphi) \mathrm{d} \omega\right\rangle}{\left\langle\int_{\Omega} \Phi_{b}(\delta \lambda) p(\varphi) \mathrm{d} \omega\right\rangle}$.

The outflow velocity then determines the relative importance of the radiative and collisional components derived from the observed total intensity.

\subsubsection{Geometry of coronal streamers}

For an axis-symmetric corona the density derived by using Eq. (3) approximates the local value on the plane of the sky. During solar minimum, the outer corona is axis-symmetric since it approximates a dipole as the streamer belt is close to the equator. This technique, however, can also be applied when the streamer is symmetric with respect to a plane parallel to the equator in a limited longitudinal interval. Since the radiative emission is linearly proportional to density, the local scale height that measures the $1 / e$ fall in density can be assumed to represent the portion of the line of sight that is dominant when determining the observed intensity of the radiative component. The relevant region is then confined to a longitudinal range on the order of $\pm \Delta \psi \sim \operatorname{arctn} \frac{H}{r}$, where $H(r)$ is the scale-height and $r$ the heliodistance. The collisional component proportional to $n^{2}$ falls even more rapidly than the radiative component; therefore, the spatial scale set for the radiative component also applies to the collisional one. Angle $\Delta \psi$ is $\sim 6$ degrees at $1.65 R_{\odot}$ and $\sim 10$ degrees at $2.7 R_{\odot}$ with the electron density derived by Gibson et al. (1999).

The streamers selected for the present analysis are approximately symmetric to the equatorial plane. Symmetric conditions exist when the neutral line of the coronal magnetic fields, extrapolated from the Wilcox Observatory data for a radial model with the location height of the source surface at $2.5 R_{\odot}$ (http://quake.stanford.edu/ wso/coronal.html), is running close to the equator (e.g., $< \pm 5$ degrees) at the streamer Carrington longitude. Only the streamer observed on August 22, 1996 is not equatorial, but in this case the tilt of the streamer symmetry plane relative to the equatorial plane is roughly \pm 5 degrees in a longitudinal range $\pm \Delta \psi \sim 10$ degrees at $2.7 R_{\odot}$. Hence, the selected quiescent streamers can be reasonably assumed as either axis-symmetric or quasi-symmetric, relative to a plane parallel to the line-of-sight in the longitude range where the coronal emission is predominantly coming from.

\subsubsection{Ion velocity distribution}

As previously discussed, resonant scattering depends on the absorption profile in the direction of incident radiation, which can be expressed in terms of kinetic temperature $T_{\mathrm{k}}$ in this direction. Inside streamers, either the plasma is static, or low speed outflows can be present as suggested by Noci et al. (1997). As a result, the velocity distribution of coronal ions is well represented by an isotropic Gaussian function with width inferred from the observed line broadening. In the dim regions adjacent to the streamer, we assume a bi-maxwellian velocity distribution. Since these regions are affected by enhanced kinetic temperatures (Antonucci et al. 1997a,b), an anisotropic distribution, as observed in the core of polar coronal holes where kinetic temperatures are extremely high (e.g., Antonucci et al. 1997a; Kohl et al. 1998; Dodero et al. 1998; Cranmer et al. 1999; Antonucci et al. 2000a, 2004), is presumed to be more appropriate. Just outside the streamer, however, the anisotropy ratio (if less than unity) has to be much lower than in the polar regions, since line broadenings are not as large as at the poles. In this case therefore, the assumption of anisotropy is not as crucial for studying the expansion of the coronal hole plasma as at the poles. To analyze the dim regions we then assume the observed kinetic temperature $T_{\mathrm{k}, \text { l.o.s. }}=T_{\mathrm{k}, \mathrm{obs}}$ in the plane perpendicular to the radial direction and equal to the electron temperature $T_{\mathrm{k}, \mathrm{r}}=T_{\mathrm{e}}$ along the radial radiation.

\subsubsection{Electron temperature}

As electron temperature $T_{\mathrm{e}}$ of a solar minimum streamer we assume the one derived by Gibson et al. (1999) (Fig. 3, dashed line), which in turn assumes a single temperature for electrons and protons, a condition verified for static plasma. In this case the electron/proton thermal equilibrium is established in 
a period on the order of $10^{4} \mathrm{~s}$ (from $\sim 5 \times 10^{3} \mathrm{~s}$ at $1.6 R_{\odot}$ to $\sim 7 \times 10^{4} \mathrm{~s}$ at $3.5 R_{\odot}$ ). The expected outflow velocities are sufficiently low above the streamer cusp to imply that electron/proton equilibrium is still valid. The curve derived by Gibson et al. at $2.7 R_{\odot}$ is consistent with the value obtained by Fineschi et al. $(1998), T_{\mathrm{e}}=(1.1 \pm 0.3) \times 10^{6} \mathrm{~K}$, by measuring with UVCS the profile of the electron scattered HI Ly $\alpha$ at $1216 \AA$ in an equatorial streamer.

It is reasonable to expect that the temperature in the dim regions along the streamer boundary falls between the temperatures of the pole and the streamer. Since at $1.5 R_{\odot}$ these two values do not differ more than a factor 2 , we then assume the same temperature measured at the poles for the regions adjacent to the streamers, and extrapolate it to the extended corona as shown in Fig. 3 (dotted line). According to David et al. (1998), the polar hole temperature never exceeds $1 \times 10^{6} \mathrm{~K}$ and declines toward the interplanetary value above $1.3 R_{\odot}$. Recently, these results were confirmed by analysis of the intensities of Fe VIII and Fe XIII lines (Ben El Hadj 2004) emitted from the same polar hole studied by David et al. The Fe line analysis fully accounts for departure from ionization equilibrium due to the coronal hole's plasma expansion. The diagnostics used to derive electron density and outflow velocity is independent of the ionization fraction. Electron temperature, therefore, influences only the collisional coefficient of line excitation and the radial profile of the absorbing ions in the case of bi-maxwellian velocity distribution. In any case, the modest excursion of electron temperature found in the extended corona means that the results of electron density and outflow velocity are fairly independent of its value.

\subsubsection{Magnetic topology}

The streamer plasma confined by closed magnetic field lines is studied assuming static conditions. However, the analysis has also been performed for dynamic conditions with the intent of identifying the site where slow wind forms along the streamer axis. In the region outside the streamer border where the field lines are likely to be open, the results are derived in the dynamic case.

The electron density and ion outflow velocity are inferred from the $n_{\mathrm{e}}(w)$ curves (Eq. (3)) derived from the observed OVI doublet intensities, as long as a constraint on mass flux conservation is applied. The electron flux can thus be extrapolated to $1 \mathrm{AU}\left(n_{\mathrm{e}} \times w, w\right)_{1 \mathrm{AU}}$, by assuming the geometry of the flow tube connecting the coronal region to the heliosphere and by applying the continuity equation in the flow tube:

$w(r) n_{\mathrm{e}}(r) A(r)=$ const.

Intersection of the extrapolated curve with the electron flux observed "in situ" in the slow wind streams with Ulysses instrumentation during the activity minimum 1996-1997 (3.5 × $10^{8} \mathrm{~cm}^{-2} \mathrm{~s}^{-1}$, McComas et al. 2000) then determines the solutions for the coronal density $n_{\mathrm{e}}$ and outflow velocity $w$ of the coronal plasma. In order to assign an appropriate geometry to the flow tube running along the boundaries of the large solar minimum coronal streamers, in the absence of any direct measurements of coronal magnetic fields, we assume a simple

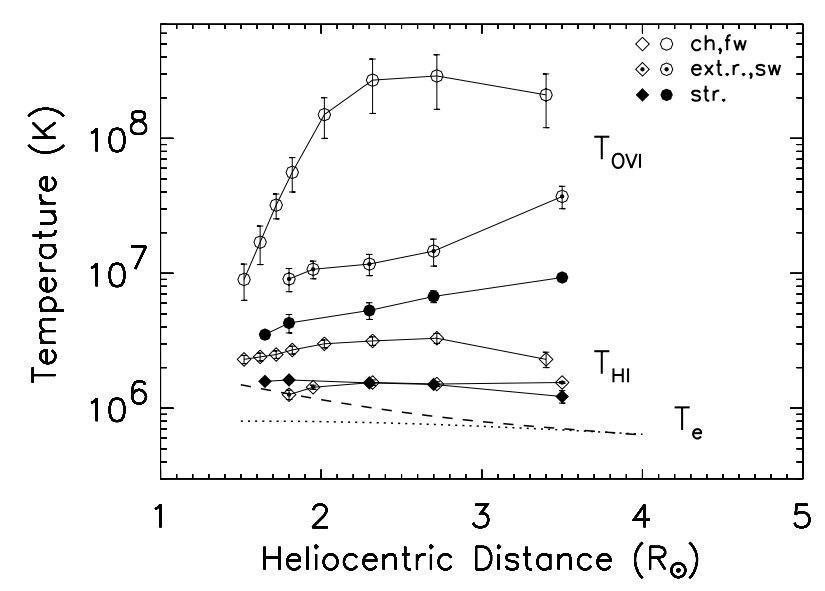

Fig. 3. Kinetic temperature of the O VI ions as a function of heliodistance for streamers (full dots), for regions external to the streamer boundary (circle-dots) and for coronal holes (open circles, from the December 31, 1997 and January 1, 1998 observations; Antonucci 1999). Kinetic temperature of the HI atoms for streamers (full diamonds), for regions external to the streamer boundary (diamond-dots) and for coronal holes (open diamonds; Antonucci 1999). The values are compared with the streamer electron temperature of Gibson et al. (1999) (dashed line) and with the coronal hole electron temperature extrapolated from the David et al. (1998) measurements obtained in the inner corona (dotted line).

magnetic model to illustrate the quiet Sun conditions (Wang \& Sheeley 1990). The coronal field is calculated for a dipolar photospheric distribution in the potential approximation. The source surface, where the field lines become radial, is set at a radius $2.5 R_{\odot}$. The model is then modified to account for an equatorial current sheet above the source surface to make it compatible with the existence of the heliospheric current sheet (see Fig. 4 by Wang \& Sheeley 1990). The inner boundary of the current sheet is then set at $2.5 R_{\odot}$. In this model the magnetic tube expansion factor is defined as

$F\left(r, \theta_{0}\right)=\left(\frac{r_{0}}{r}\right)^{2}\left(\frac{B_{r}\left(r_{0}, \theta_{0}\right)}{B_{r}(r, \theta)}\right)$

where $B_{r}\left(r_{0}, \theta\right)=\cos ^{n} \theta$ is the radial component of the photospheric field, $\theta$ the co-latitudinal position of the flux tube, and $n$ a flux concentration index, assumed $n=7$ to approximate the expansion of the field lines observed in polar holes near sunspot minimum (e.g. Munro \& Jackson 1977; Dobryzcka et al. 1999). In this model the flux tube formed by the open magnetic field lines adjacent to the streamer exhibits an extremely large expansion factor near the source surface as suggested by the visible light and UV coronal images. In this analysis, the regions outside the observed $1 / e$-intensity boundary are 15 degrees wide on the average. Due to the rapid decrease in intensity outside streamers, however, a thinner layer close to the boundary is predominant in the emission, making the relevant flux tube narrower than the observed geometrical one. We then assume that the boundary lies close to the first open field lines outlining the current sheet, and characterize the layers immediately outside the boundary by the expansion factor computed for the first open field lines outlining the current sheet, with a maximum value $F\left(r, \theta_{0}\right)_{\max } \sim 13.5$ near $2.5 R_{\odot}$ and a heliospheric 

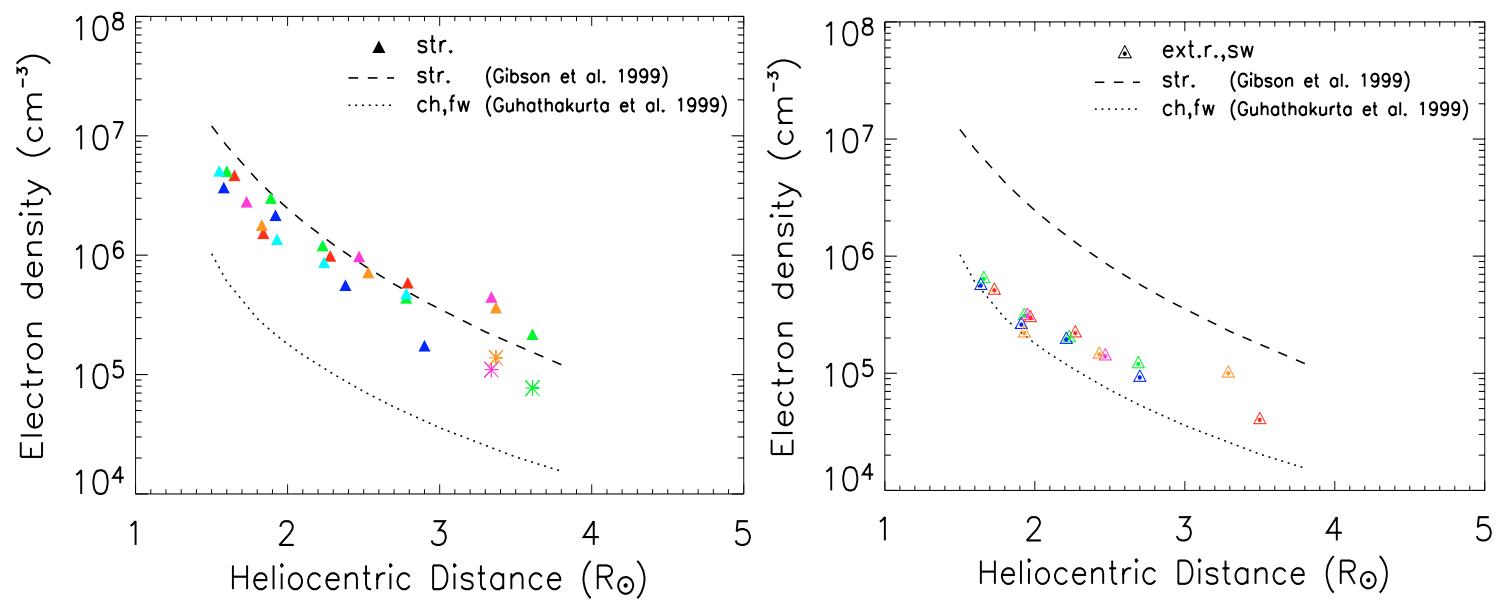

Fig. 4. Electron density as a function of height for the individual streamers listed in Table 1: different colours indicate different streamers. The results are compared with the streamer electron density derived by Gibson et al. (1999) (dashed line) and by Guhathakurta et al. (1999) for coronal holes (dotted line). In the left panel, full triangles indicate the streamer results obtained for a static plasma and the asterisks for an expanding plasma. The right panel shows the results for the region outside the streamer boundary.

value $\sim 5$ (Fig. 4b by Wang \& Sheeley 1990). For comparison, the expansion factor calculated for the core of the coronal hole is $\sim 5$ at the same coronal height. The flux tube geometry and relative expansion factor used in the analysis are derived with the code provided by Dr. Y. H. Wang.

\section{Data analysis results}

\subsection{Oxygen ion kinetic temperature in streamers and adjacent regions}

The coronal kinetic temperatures of oxygen ions inferred from the width of the O VI 1032 line are plotted in Fig. 3. The results are obtained for the six individual streamers and then averaged. The figure statistically confirms the existence of a significant variation in oxygen kinetic temperature $T_{\mathrm{k}, \mathrm{O}} \mathrm{VI}$ across the streamer boundary out to $3.5 R_{\odot}$ (e.g. Antonucci et al. 1997a,b; Dobryzcka et al. 1999) and shows an increase of this quantity with height. In streamers, $T_{\mathrm{k}, \mathrm{O}} \mathrm{VI}$ increases from $3.5 \pm 0.2 \times 10^{6} \mathrm{~K}$ at $1.65 R_{\odot}$ to $9.3 \pm 0.1 \times 10^{6} \mathrm{~K}$ at $3.5 R_{\odot}$ (full dots). The error bars result from propagation of the statistical uncertainties of the observed O VI 1032 line intensity. The low error bars ensure that the plasma conditions are similar in the six streamers analyzed. In the dim regions outside streamers, $T_{\mathrm{k}, \mathrm{O}} \mathrm{VI}$ is higher than inside streamers and increases from $0.9 \pm 0.2 \times 10^{7} \mathrm{~K}$ at $1.8 R_{\odot}$ to $3.7 \pm 0.7 \times 10^{7} \mathrm{~K}$ at $3.5 R_{\odot}$ (circle-dots). The increase across the boundary is larger at higher altitudes by a factor $\sim 2$ and $\sim 4$, at $1.8 R_{\odot}$ and $3.5 R_{\odot}$, respectively. Since the coronal electron temperature lies between the streamer and coronal hole values (dashed and dotted line, respectively, Fig. 3), the derived kinetic temperatures imply an oxygen velocity distribution broader than expected for thermal equilibrium, and this effect is significantly enhanced outside streamers. In Fig. 3 the oxygen kinetic temperature results are compared to those obtained at the poles (open dots in Fig. 3, Antonucci 1999). The latitudinal increase in kinetic temperature suggests that the ion velocity distribution anisotropy across the magnetic field observed at the poles (e.g. Kohl et al. 1998; Dodero et al. 1998; Cranmer et al. 1999; Antonucci et al. 2000a) may also exist at lower latitude in the corona where field lines are open. For this reason, we assume a bi-maxwellian distribution in the analysis of the regions outside the streamer boundary (Sect. 3.2.2). The velocity distribution of the hydrogen atoms, reflecting that of protons, is also shown in Fig. 3. In streamers, the kinetic temperature of the hydrogen atoms, as derived from the HI Lyman $\alpha$ line broadenings, is higher than outside streamers below $2 R_{\odot}$ (full diamonds in Fig. 3), as pointed out by Zangrilli et al. (1999). The opposite, however, occurs in the outer corona at about $3.5 R_{\odot}$, where the hydrogen kinetic temperature shows instead a tendency to increase outside the boundary. As in the case of oxygen, the highest hydrogen temperature is found at the poles (open diamonds in Fig. 3). The kinetic temperature of hydrogen remains, in any case, two orders of magnitude lower than that of oxygen.

\subsection{Density and outflow velocity results}

In this section we discuss the electron density and outflow velocity results obtained for the two regions separated by the streamer boundary. These results are obtained individually for each streamer and then averaged.

\subsubsection{Density results}

Density of individual streamers, shown in the left panel of Fig. 4, is derived from analysis of the OVI doublet assuming magnetically confined plasma and isotropic oxygen velocity distribution. Figure 5 and Table 3 report the average results. Below $3.5 R_{\odot}$ density (full triangles) is generally lower than that inferred from the visible light observations (dashed line, Gibson et al. 1999). This tendency is also maintained further out, if the hypothesis of static conditions is relaxed by allowing for plasma expansion (asterisks in Figs. 4 and 5). If the analysis is performed assuming expanding plasma at all heights, unreasonably low values (of the same order as densities found 
Table 3. Average electron density $n_{\mathrm{e}}$ inside streamers and in the dim regions outside the streamer boundary.

\begin{tabular}{cc|cc}
\hline \hline \multicolumn{2}{c|}{ Streamer } & \multicolumn{2}{c}{ Streamer adjacent region } \\
\hline $\begin{array}{c}\text { Heliodistance } \\
\left(R_{\odot}\right)\end{array}$ & $\begin{array}{c}n_{\mathrm{e}} \\
\left(\mathrm{cm}^{-3}\right)\end{array}$ & $\begin{array}{c}\text { Heliodistance } \\
\left(R_{\odot}\right)\end{array}$ & $\begin{array}{c}n_{\mathrm{e}} \\
\left(\mathrm{cm}^{-3}\right)\end{array}$ \\
\hline 1.65 & $4.1 \pm 0.4 \times 10^{6}$ & 1.80 & $5.7 \pm 0.6 \times 10^{5}$ \\
1.80 & $1.9 \pm 0.3 \times 10^{6}$ & 1.95 & $2.8 \pm 0.2 \times 10^{5}$ \\
2.30 & $8.5 \pm 0.6 \times 10^{5}$ & 2.30 & $1.8 \pm 0.3 \times 10^{5}$ \\
2.70 & $4.0 \pm 0.6 \times 10^{5}$ & 2.70 & $1.1 \pm 0.2 \times 10^{5}$ \\
3.50 & $3.3 \pm 0.6 \times 10^{5}$ & 3.50 & $7.0 \pm 1.8 \times 10^{4}$ \\
$3.50^{1}$ & $1.1 \pm 0.6 \times 10^{5}$ & & \\
\hline
\end{tabular}

${ }^{1}$ In this case, the corona is allowed to expand.

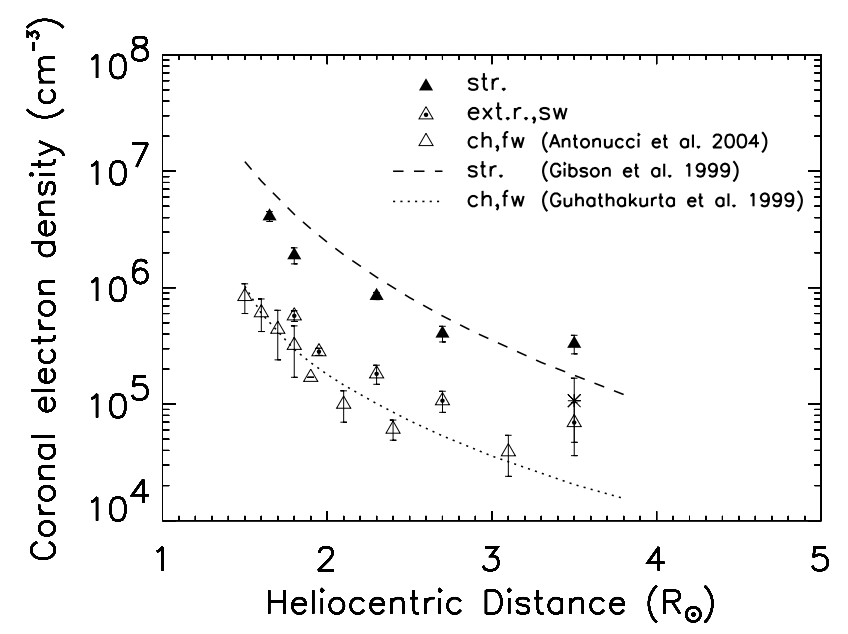

Fig. 5. Plasma electron density as a function of heliocentric distance (in solar radii), averaged over the sample of observations, for: a) streamers (full triangles, at $3.5 R_{\odot}$ the value of electron density derived by allowing coronal expansion, is denoted by an asterisk); b) dim regions outside the streamer boundary (triangle-dots). The electron density derived with the same technique for a polar coronal hole (Antonucci et al. 2004, open triangles), from visible light observations for streamers (Gibson et al. 1999, dashed line) and for coronal holes (Guhathakurta et al. 1999, dotted line) are reported for comparison.

in the dim external regions) are obtained below $3.5 R_{\odot}$. At $2.3 R_{\odot}$, the present results are consistent with the density obtained by Strachan et al. (2002) along the streamer axis, whereas they are lower at higher heliodistances. The above results indicate that inside streamers, $\leq 2.7 R_{\odot}$, density values agree with closed magnetic field lines that confine the coronal plasma in the bright regions delimited by the $1 / e \mathrm{O}$ VI intensity contour line; whereas beyond $2.7 R_{\odot}$, the plasma slowly expands along the streamer axis. Hence we can set the transition to within $2.7-3.5 R_{\odot}$ between closed and open field lines in streamers.

In the coronal layers outside the streamer boundary, the plasma is allowed to flow in a flux tube with a large expansion factor (see Sect. 3.2.5). The density is obtained by intersecting the inferred electron flux as a function of the outflow velocity and extrapolated to $1 \mathrm{AU}$ with the value measured "in situ". An example of this method is given in Fig. 6, where for instance at $1.9 R_{\odot}$ two solutions are found: the first consistent with a static plasma, within the statistical uncertainty, the second consistent with an expansion at a velocity of about $90 \mathrm{~km} \mathrm{~s}^{-1}$. Also in this case, the regions adjacent to streamers are analyzed individually (right panel of Fig. 4) and then the results are averaged (triangle-dots, Fig. 5, Table 3). The density used by Strachan et al. (2002) at $2.3 R_{\odot}$ in the vicinity of the streamer is consistent with the results obtained at the same height in Fig. 5. If the hypothesis of expanding plasma is dropped, unrealistically high density values are obtained, which then excludes any possibility that the plasma remains confined in these regions. In the analysis we have assumed a coronal temperature extrapolated from the coronal hole measurement of David et al. (1998); however the results remain unchanged within statistical errors, if a temperature equal to the streamer temperature is assumed. These results prove that the external layer along the streamer boundary is indeed characterized by open magnetic field lines that can allow wind flow. Thus we conclude that the $1 / e$ intensity boundary is a good approximation of the interface between closed and open field lines in the extended corona. In other words, this boundary represents the interface between coronal hole and streamer well. It follows that we can identify the dim regions around the boundary as equivalent to the far edges of the large solar minimum coronal holes, for which density values fall midway between the streamer values and those found in the core of holes (open triangles), as shown in Fig. 5. The density of the hole edge below $2 R_{\odot}$ remains very close to that of the core. This strengthens the suggestion that the open field lines running along the streamer boundary originate in the same unipolar magnetic region lying at the base of a polar coronal hole, which is approximately uniform in density. Further out in the corona, in contrast, density varies significantly from the core to the edges of the hole. This means that the derivative of density with height is different if the flux tubes driving the solar wind originate in the core or at the edge of a coronal hole. In the first case the density falls off very rapidly, whereas close to the hole boundary it declines less rapidly and, at $3.5 R_{\odot}$, it reaches a value close to that observed above the streamer cusp. This indicates that the factor regulating the density along the 

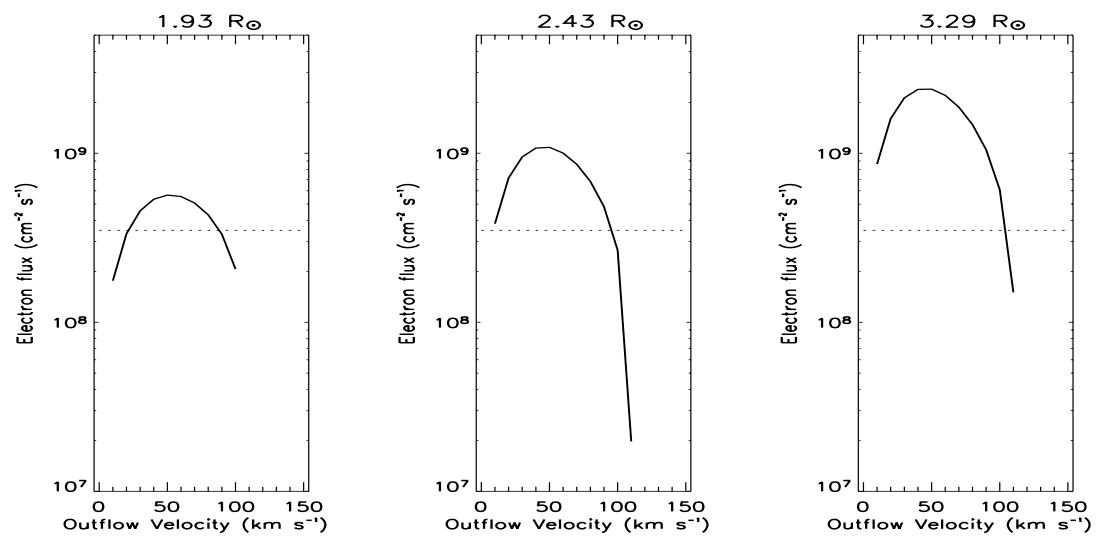

Fig. 6. Electron flux of the coronal region outside the streamer boundary observed on May 5, 1997, as a function of outflow velocity, extrapolated to $1 \mathrm{AU}$, derived for each heliocentric distance. The horizontal line corresponds to the electron flux value of $3.5 \times 10^{8} \mathrm{~cm}^{-2} \mathrm{~s}^{-1}$ obtained by Ulysses measurements during the minimum of solar activity (McComas et al. 2000).

flux tubes is the geometry of the magnetic tube, that is, its expansion factor which is lower in the core of coronal holes and higher close to the hole-streamer boundary interface.

\subsubsection{Outflow velocity results}

In the dim regions that lie in the immediate vicinity of streamers, identified as the far edges of polar coronal holes, the speed ranges approximately from $90 \mathrm{~km} \mathrm{~s}^{-1}$ at $1.8 R_{\odot}$ to $110 \mathrm{~km} \mathrm{~s}^{-1}$ at $3.5 R_{\odot}$. Flows are already formed when observed at the lower limit of the UVCS field of view. Above the streamer cusp, the plasma expands at $90 \mathrm{~km} \mathrm{~s}^{-1}$ (Table 4). Outflow velocities derived by applying the magnetic topology proposed by Wang and Sheeley are higher than those previously derived using an average coronal hole expansion factor (equal to 7, Abbo $\&$ Antonucci 2002a). We then expect that when assuming a more accurate flux tube geometry for the region adjacent to streamers, analysis of Doppler dimming becomes more sensitive to lower velocities than the present one. Velocity values derived for the oxygen component of the solar wind are consistent with the speed of the small coronal inhomogeneities detaching from the streamer cusp, as observed with LASCO, which does not exceed $100 \mathrm{~km} \mathrm{~s}^{-1}$ at 3-4 $R_{\odot}$ (Wang et al. 2000). The oxygen outflow speed is representative of the proton outflow speed, since the expansion time locally does not exceed the proton/oxygen thermal equilibrium time. The inferred coronal outflow velocity might be underestimated below $2.5 R_{\odot}$ due to deviations from radial expansion, since Doppler dimming measures only the radial component of outflows. Expansion is expected, however, to become predominantly radial with increasing heliodistance (see Fig. 4 by Wang \& Sheeley 1990). Therefore at approximately $3.5 R_{\odot}$ where field lines are close to radial, the outflow velocity should be accurately derived with Doppler dimming techniques. The fact that at this height the speed is on the order of $100 \mathrm{~km} \mathrm{~s}^{-1}$ ensures lower values lower down as was indeed observed (see Table 4), provided that the velocity gradually increases along the flux tube. When considering that the fast wind expands much more rapidly in the core of a coronal hole, with a speed that varies from about $100 \mathrm{~km} \mathrm{~s}^{-1}$ to $400 \mathrm{~km} \mathrm{~s}^{-1}$ between $1.8 R_{\odot}$ and $3.5 R_{\odot}$ (e.g. Kohl et al. 1998; Cranmer et al. 1999; Antonucci 1999; Antonucci et al. 2000b, 2004), the value of approximately $100 \mathrm{~km} \mathrm{~s}^{-1}$ found near streamers clearly indicates a regime of slow flow. The observed slow wind, with speed far from the asymptotic heliospheric value of approximately $400 \mathrm{~km} \mathrm{~s}^{-1}$, is then still in a region of acceleration. The present analysis suggests the existence of two components in the coronal slow wind. The wind adjacent to the streamer boundary flows along the flux tube formed by the open field lines delimiting the layer of the heliospheric current sheet. An additional component of slow wind forms above the streamer cusp roughly corresponding to the heliospheric current sheet formation and tends to flow more slowly than in the layers along the streamer boundary. The two components, however, have very similar properties at $3.5 R_{\odot}$. The component of the slow wind streaming along the boundary originates in the polar coronal hole, and is guided by open field lines that are characterized by a much higher expansion factor at the edges of a hole than in the core, according to the Wang and Sheeley model; whereas the fast wind flows along the field lines emerging at the center of a solar minimum coronal hole. Slow and fast winds thus have similar initial density conditions. At lower heights, e.g. $1.8 R_{\odot}$, outflow velocity varies from the edge to the core of the coronal hole to a lesser degree than do the electron density and the ion kinetic temperature. Significant differences arise, however, further out. At 3.5 solar radii, the outflow velocity (e.g. Antonucci et al. 2000a, 2004), kinetic temperature of the ions, and electron density (Figs. 3 and 5) vary significantly from the edge to the core of coronal holes, thus showing a clear dependence on latitude of the height-derivative of these quantities. This translates into a dependence on the magnetic topology of the extended corona.

\section{Discussion}

This analysis of electron density and ion outflow velocity in the streamer belt of the minimum corona - based on the O VI 1032 and 1038 emission observed with the UVCS and performed by assuming that the coronal magnetic topology can be illustrated by the model of Wang \& Sheeley (1990) - leads to the 
Table 4. Outflow velocity derived for the streamer and for the regions external to streamers, as a function of height.

\begin{tabular}{cc|cc}
\hline \hline \multicolumn{2}{c|}{ Streamer } & \multicolumn{2}{c}{ Streamer adjacent region } \\
\hline $\begin{array}{c}\text { Heliodistance } \\
\left(R_{\odot}\right)\end{array}$ & Outflow velocity & $\begin{array}{c}\text { Heliodistance } \\
\left(\mathrm{km} \mathrm{s}^{-1}\right)\end{array}$ & $\begin{array}{c}\text { Outflow velocity } \\
\left(R_{\odot}\right)\end{array}$ \\
\hline 1.65 & - & 1.80 & $92 \pm 4$ \\
1.80 & - & 1.95 & $87 \pm 5$ \\
2.30 & - & 2.30 & $87 \pm 9$ \\
2.70 & - & 2.70 & $93 \pm 7$ \\
3.50 & $94 \pm 5$ & 3.50 & $113 \pm 16$ \\
\hline
\end{tabular}

conclusion that the dim regions in the immediate vicinity of the streamer boundaries are sites of outflows at low velocities and therefore likely sources of the slow wind. In addition, coronal expansion at low speed is observed along the streamer beyond $2.7 R_{\odot}$, presumably where the transition between closed and open magnetic field lines takes place and the interplanetary current sheet forms.

The slow wind observed above the streamer cusp might be due to merging of the wind flowing along the streamer boundaries. Alternatively it might be of a different nature than that flowing along the streamer boundary. For instance, this slow wind component might originate either in flux tubes between sub-streamers (Noci et al. 1997) or in the regions between the core of the streamer, delimited by closed field lines and the first open field lines, namely the streamer stalks, as suggested by Raymond et al. (1997). According to the Raymond et al. hypothesis, these areas, bright in oxygen when observed in quiet equatorial streamers, might also be slow wind sources. Due to their brightness, however, such regions lie within the $1 / e$ boundaries and are not resolved in this analysis of streamers, since the emission inside the boundaries is averaged. A two-component slow wind is inferred by Wang et al. (2000) by studying the flow of small inhomogeneities, or coronal blobs, forming at the streamer cusp and moving along the plasma/current sheet (Sheeley et al. 1997). The speed of the slow wind observed above the streamer cusp is consistent with the speed of coronal blobs.

That the regions just outside the streamer boundary are sites of plasma outflows proves that they are characterized by open field lines and, therefore, can be identified with the far edges of the large polar coronal holes existing during solar minimum. Hence, the boundary defined in terms of the $1 / e$ emission of the $\mathrm{O}$ VI lines and assumed in the present analysis is a fairly good definition of the transition between open and closed magnetic field lines. Oxygen ion kinetic temperature, outflow velocity, and electron density results, all point to a gradual variation of the physical and dynamical conditions of a coronal hole from the edge, limited by the equatorial streamer boundary, to the polar core. The gradient in latitude of these quantities is, however, rapidly increasing with height, at least up to $2.3-2.5 R_{\odot}$. It is to be noted that fast and slow wind share approximately the same density lower in the corona. In this scenario the sharpest transition in the extended corona is indeed observed to correspond to the streamer border. This contrasts with the in situ measurements of speed, charge-state composition, and elemental composition of the heliospheric solar wind (e.g., Von Steiger et al. 2000), all of which change abruptly from slow to fast wind streams, thus defining a sharp boundary between the two wind regimes. This analysis thus indicates that the sharp heliospheric interface between fast and slow wind is not detectable at coronal level and is then most likely formed further out when the wind propagates through the heliosphere.

The gradual variation of the physical parameters in the corona characterized by open magnetic field lines points to a correlation of plasma conditions and magnetic topology and, in particular, to a correlation of plasma parameters and the expansion factor of the flux tubes that is monotonically changing from the hole center to the edges below $2.5 R_{\odot}$. Therefore, the most likely interpretation is that divergence of the open field lines is regulating the parameters of the expanding coronal plasma. The faster wind would then originate in the center of the unipolar magnetic region at the base of the coronal hole, where the field lines are less divergent, and the slower wind forms at the edges of the unipolar region, where field lines become highly divergent in the outer corona. All of this leads us to suggest that the physical conditions in the open field line regions of the outer corona depend on the local magnetic topology, which agrees with the lack of clear evidence of the sharply abrupt variation between fast and slow streams found in the heliosphere.

The enhanced broadening of oxygen ion velocity distributions across the magnetic field at the edges of coronal holes is very likely of a similar nature to that observed in the polar hole regions. We suggest to interpret this effect as a signature of energy deposition, although to a lesser degree than in the center of coronal holes. The same mechanism might, therefore, act in the core and at the edges of a coronal hole. We suggest in this case, too, that along this line there is energy dissipation through ion cyclotron resonance of high-frequency Alfvén waves, that is, the process proposed as the mechanism that heats the ions preferentially across the magnetic field and thus causes the fast solar wind acceleration in the core of coronal holes (Kohl et al. 1998; Cranmer et al. 1999). If this is the case, the present results then point to a gradually increasing energy deposition from the streamer boundary to the core of polar holes, accompanied by a gradual increase in velocity. It is interesting to note that also inside streamers, where plasma is mainly confined by the magnetic field, we observe an increase in oxygen kinetic 
temperature above that acquired by protons and electrons, although the effect is lower than in the open magnetic field regions (see Frazin et al. 2003). This would point to the fact that is also active inside streamers energy deposition, although at a much lower level than in coronal holes; in this case energy is not transformed into acceleration of the oxygen component of the solar wind, as occurs in open field line regions due to the field topology.

This scenario agrees with the suggestion of Wang \& Sheeley (1990) that the velocity of solar wind is regulated by the magnetic topology of the solar wind, which flows more rapidly in flux tubes with a smaller expansion factor. This leads to a fast wind in the less divergent flux tubes of the core of a polar coronal hole, where the expansion factor is low, and to a much slower wind in the highly divergent flux tubes surrounding the streamer closed field lines, where the expansion factor is likely to be about 3 times higher. However, the present results indicate as well that the ion velocity distribution across the magnetic field lines also varies according to the field's topology. If interpreted in terms of ion acceleration across the field lines - due to energy dissipation through, for instance, ion cyclotron resonance of high-frequency Alfvén waves - the implication is that this effect is more efficient in less divergent flux tubes, leading to a higher acceleration in these zones. Therefore not only is particle flux regulated by the field geometry, but the geometry is also regulating the energy dissipation due to the high-frequency waves that have been suggested to be driving the fast wind.

Acknowledgements. We thank Dr. Y. H. Wang for valuable discussions and for providing the code to compute the coronal magnetic field, and Ms. M. Mierla for providing the LASCO-C1 images. We thank Dr. S. Giordano for his analysis of the December 31, 1997 and January 1, 1998 data. Work on the streamer-coronal hole boundary has been stimulated by participation in the International Space Science Institute (Bern) Working Group "Tracing coronal hole boundaries into the solar wind", held in 2000-2001. UVCS is a joint project of the National Aeronautics and Space Administration (NASA), the Agenzia Spaziale Italiana (ASI) and Swiss Founding Agencies. The authors wish to acknowledge the support of the Agenzia Spaziale Italiana, ASI, through contracts ASI/IR/084/02 and ASI/IR/125/01 and of the Ministero dell'Università e della Ricerca Scientifica e Tecnologica through the grant COFIN-2001.

\section{References}

Abbo, L., \& Antonucci, E. 2002a, From Solar Min to Max: Half a Solar Cycle with SOHO, ESA-SP, 508, 477

Abbo, L., \& Antonucci, E. 2002b, SOLSPA: The Second Solar Cycle and Space Weather Euroconference, ESA-SP, 477, 323

Abbo, L. 2002, Ph.D. Thesis, Identification of the coronal sources of the slow solar wind, Università degli Studi di Torino

Abbo, L., Antonucci, E., Dodero, M. A., \& Benna, C. 2003, Proceedings Solar Wind 10, AIP, 679, 238

Antonucci, E., Noci, G., Kohl, J. L., et al. 1997a, ASP Ser., 118, 273

Antonucci, E., Giordano, S., Benna, C., et al. 1997b, The Corona and Solar Wind near Minimum Activity, ESA-SP, 404, 175

Antonucci, E. 1998, A Crossroads for European Solar and Heliospheric Physics, ESA-SP, 417, 25

Antonucci, E. 1999, Plasma Dynamics and Diagnostics in the Solar Transition Region and Corona, ESA-SP, 446, 53
Antonucci, E., Dodero, M. A., \& Giordano, S. 2000a, Sol. Phys., 197, 115

Antonucci, E., Giordano, S., \& Dodero, M. A. 2000b, Adv. Space Res., 25, 1923

Antonucci, E., Dodero, M. A., Giordano, S., Krishnakumar, V., \& Noci, G. 2004, A\&A, 416, 749

Ben El Hadj, R. 2004, Ph.D. Thesis, University of Paris XI, Orsay

Cranmer, S. R., Kohl, J. L., Noci, G., et al. 1999, ApJ, 511, 481

David, C., Gabriel, A. H., Bely-Dubau, F., et al. 1998, A\&A, 336, L90

Dobrzycka, D., Cranmer, S. R., Panasyuk, A. V., Strachan, L., \& Kohl, J. L. 1999, JGR, 104, A5, 9791

Dodero, M. A., Antonucci, E., Giordano, S., \& Martin, R. 1998, Sol. Phys., 183, 77

Fineschi, S., Gardner, L. D., Kohl, J. L., Romoli, M., \& Noci, G. 1998, SPIE Proc., 3443, 67

Frazin, R. A., Cranmer, S. R., \& Kohl, J. L. 2003, ApJ, 597, 1145

Gardner, L. D., Kohl, J. L, Daigneau, P. S., et al. 1996, Proc. SPIE, 2831,2

Gibson, S. E., Fludra, A., Bagenal, F., et al. 1999, JGR, 104, 9691

Giordano, S., Antonucci, E., Benna, C., et al. 1997, Correlated Phenomena at the Sun, in the Heliosphere and in Geospace, ESA-SP, 415, 327

Giordano, S. 1999, Ph.D. Thesis, Università degli Studi di Torino

Guhathakurta, M., Fludra, A., Gibson, S. E., Biesecker, D., \& Fisher, R. 1999, JGR, 104, A5, 9801

Habbal, S. R., Woo, R., Fineschi, S., et al. 1997, ApJ, 489, L103

Ko, Y., Raymond, J. C., Li, J., et al. 2002, ApJ, 578, 979

Kopp, R. A., \& Holzer, T. E. 1976, Sol. Phys., 49, 43

Kohl, J. L., Esser, R., Gardner, L. D., et al. 1995, Sol. Phys., 162, 313

Kohl, J. L., Noci, G., Antonucci, E., et al. 1997, Sol. Phys., 175, 613

Kohl, J. L., Noci, G., Antonucci, E., et al. 1998, ApJ, 501, L127

Li, X., Habbal, S. R., Kohl, J. L., \& Noci, G. 1998, ApJ, 501, L133

Marocchi, D., Antonucci, E., \& Giordano, S. 2001, Ann. Geophys., 19,135

McComas, D. J., Bame, S. J., Barraclough, B. L., et al. 1998, Geophys. Res. Lett., 25, 1, 1

McComas, D. J., Barraclough, B. L., Funsten, H. O., et al. 2000, JGR, 105,10419

Munro, R. H., \& Jackson, B. V. 1977, ApJ, 213, 874

Noci, G., Kohl, J. L., \& Withbroe, G. L. 1987, ApJ, 315, 706

Noci, G., Kohl, J. L., Antonucci, E., et al. 1997, Fifth SOHO workshop ESA SP-404, 75

Phillips, J. L., Bame, S. J., Barnes, A., et al. 1995, Geophys. Res. Lett., 22,3301

Pneuman, G. W., \& Kopp, R. A. 1971, Sol. Phys., 18, 258

Raymond, J. C., Kohl, J. L., Noci Antonucci, E., et al. 1997, Sol. Phys., 175,645

Sheeley, N. R., Wang, Y.-M., Hawley, S. H., et al. 1997, ApJ, 484, 472

Strachan, L., Suleiman, R., Panasyuk, A. V., Biesecker, D. A., \& Kohl, J. L. 2002, ApJ, 571, 1008

Uzzo, M., Ko, Y.-K., Raymond, J. C., Wurz, P., \& Ipavich, F. M. 2003, ApJ, 585, 1062

von Steiger, R., Schwadron, N. A., Fisk, L. A., et al. 2000, JGR, 105, 27217

Wang, Y. H., \& Sheeley, N. R. 1990, ApJ, 355, 726

Wang, Y. H., Sheeley, N. R., Socker, D. G., Howard, R. A., \& Rich, N. B. 2000, JGR, 105, 25133

Wilhelm, K., Lemaire, P., Dammasch, I. E., et al. 1998, A\&A, 334, 685

Zangrilli, L., Nicolosi, P., Poletto, G., et al. 1999, A\&A, 342, 592

Zangrilli, L., Poletto, G., Nicolosi, P., Noci, G., \& Romoli, M. 2002, ApJ, 574, 477

Zurbuchen, T. H., Von Steiger, R., Riley, P., et al. 2001, AGU Spring Meeting Abstracts, 21 